
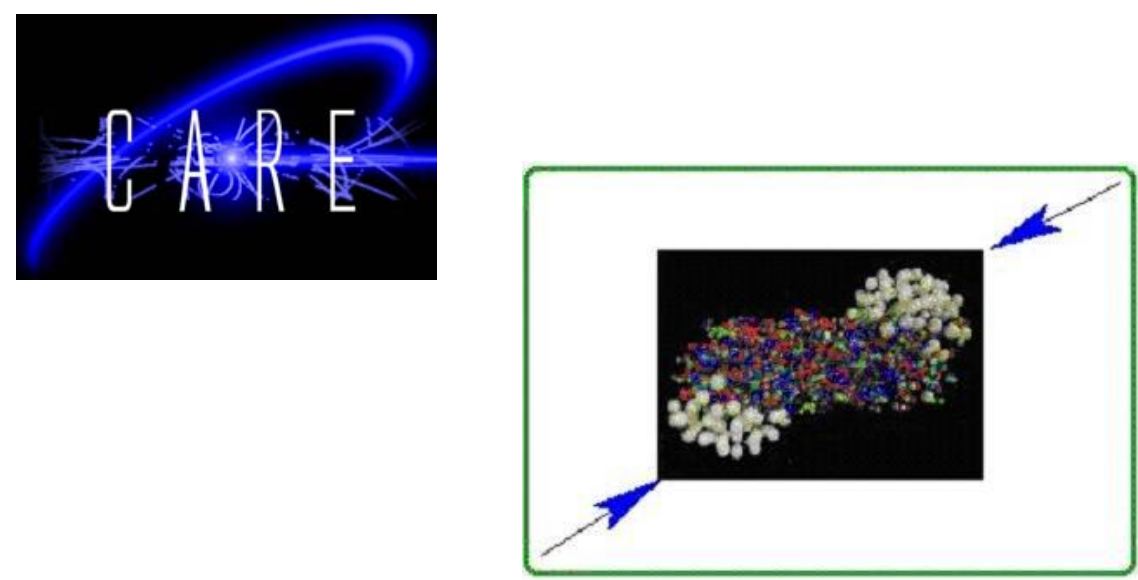

High Energy

High Intensity

Hadron Beams

\author{
PLANS FOR THE FUTURE OF PROTON ACCELERATORS AT CERN \\ R. Garoby, W. Scandale, CERN, Geneva, Switzerland
}

\begin{abstract}
The Large Hadron Collider, presently in construction at CERN, will be filled through a set of high performance proton accelerators providing the high brightness beam needed to reach the foreseen luminosity. Although this difficult project has top priority and uses most of the CERN resources, it is nevertheless time investigating improvements of the proton accelerator complex for physical cases beyond the LHC expectations. The needs of multiple physics communities have to be taken into account, as well as the necessity of consolidating the installations while keeping high reliability. This paper starts from the analysis and proposals made by the "High Intensity Proton" (HIP) working group [1, 2] to improve the performances of the PS and the SPS complex and better match the users requests in a staged scenario at short and medium term, and complement it, addressing the main possibilities beyond that horizon.
\end{abstract}

Contribution to the High Intensity Frontier Workshop (HIF04), Isola d’Elba, Italy

Work supported by the European Community-Research Infrastructure Activity under the FP6 "Structuring the European Research Area" programme (CARE, contract number RII3-CT2003-506395). 


\title{
PLANS FOR THE FUTURE OF PROTON ACCELERATORS AT CERN
}

\author{
R. Garoby, W. Scandale, CERN, Geneva, Switzerland
}

\begin{abstract}
The Large Hadron Collider, presently in construction at CERN, will be filled through a set of high performance proton accelerators providing the high brightness beam needed to reach the foreseen luminosity. Although this difficult project has top priority and uses most of the CERN resources, it is nevertheless time investigating improvements of the proton accelerator complex for physical cases beyond the LHC expectations. The needs of multiple physics communities have to be taken into account, as well as the necessity of consolidating the installations while keeping high reliability. This paper starts from the analysis and proposals made by the "High Intensity Proton” (HIP) working group [1, 2] to improve the performances of the PS and the SPS complex and better match the users requests in a staged scenario at short and medium term, and complement it, addressing the main possibilities beyond that horizon.
\end{abstract}

\section{OUTCOME OF THE HIP WG}

\section{Physics requests}

The HIP working group has recently established a list of requests from the physics teams already working at CERN and recommended a staged path for the upgrade of the proton accelerators [1, 2]. The needs of LHC, COMPASS, neutrino and radio-active ion beam physics have been taken into account. For the other present users, i.e. AD, PS East area and nToF, the assumption has been that their requirements do not significantly modify the choice, and that every upgrade scenario would be compatible.

In terms of schedule and resources, the users' requests fall into three main categories: (i) the short term, "low" (ideally zero) cost demands, which match the present commitments of CERN and belong to the approved physics programme, (ii) the medium term, "medium" cost requests, which correspond to modest and progressive increases of performance for the present experiments, (iii) the long term, "high" cost wishes, which are linked to major equipment upgrades and to new experiments suggested for integration inside the future physics programme of CERN. These are summarised in Table 1.

\section{Accelerators needs}

The Linac2, PSB, PS and SPS have been built more than 35 years ago. Although a significant fraction of their equipment has been renovated, the most expensive ones are the oldest and show weaknesses. This has been aggravated by the reduction or even by the suppression of preventive maintenance due to the lack of resources during the past years. Therefore consolidation is essential in the near future and, in the medium term, the replacement of these accelerators deserves serious consideration. Moreover, the reduction of beam losses is a major issue in order to minimize the material irradiation for improved reliability and even more important the dose taken by the personnel during maintenance.

\begin{tabular}{|c|c|c|c|}
\hline \multirow[t]{2}{*}{ User } & \multirow{2}{*}{$\begin{array}{c}\text { CERN } \\
\text { commitment* } \\
\text { Short term }\end{array}$} & \multicolumn{2}{|c|}{ Users' wishes } \\
\hline & & Medium term & $\begin{array}{c}\text { Long term } \\
{[>2014]}\end{array}$ \\
\hline LHC & $\begin{array}{c}\text { Nom. } \\
\text { luminosity } \\
10^{34} \mathrm{~cm}^{-2} \mathrm{~s}^{-1} \\
\end{array}$ & $\begin{array}{c}\text { Ultimate } \\
\text { luminosity } \\
2.3 \square 10^{34} \mathrm{~cm}^{-2} \mathrm{~s}^{-1}\end{array}$ & $\begin{array}{l}\text { Luminosity } \\
\text { upgrade } \\
\text { (tenfold) }\end{array}$ \\
\hline $\begin{array}{c}\text { SFT } \\
\text { (COMPASS) }\end{array}$ & $\begin{array}{c}4.3 \square 10^{5} \\
\text { spills/year? }\end{array}$ & $\begin{array}{c}7.2 \square 10^{5} \\
\text { spills/year }\end{array}$ & \\
\hline CNGS & $4.5 \square 10^{19} \mathrm{p} /$ year & $\begin{array}{c}\text { Upgrade } \\
\sim \square 2\end{array}$ & \\
\hline ISOLDE & $1.92 \mu \mathrm{A}^{* *}$ & $\begin{array}{c}\text { Upgrade } \\
\sim \square 5\end{array}$ & \\
\hline $\begin{array}{c}\text { Future } v \\
\text { beams }\end{array}$ & & & $\begin{array}{c}>2 \mathrm{GeV} \text {, } \\
4 \mathrm{MW}\end{array}$ \\
\hline EURISOL & & & $\begin{array}{c}>1-2 \mathrm{GeV}, \\
5 \mathrm{MW}\end{array}$ \\
\hline
\end{tabular}

* Reference value for analysis.

** 1350 pulses/hour - 3.2 $\square 10^{13}$ protons per pulse (ppp).

\section{Basic assessment}

The analysis of the proton flux available to the users starts in 2007 corresponding to the first year of LHC operation, under the following assumptions:

\section{- Accelerators operating time per year}

o PS: 5400 h (without setting-up)

o SPS/LHC: $4700 \mathrm{~h}$ (without setting-up)

o SPS in LHC filling mode: $15 \%$ (5\%) of the time

o SPS in LHC pilot mode: $35 \%$ (10\%) of the time

o SPS in CNGS\&SFT mode: 50\% (85\%) of the time

- Availability

o PS \& PSB: $90 \%$

o SPS : $80 \%$

- Beam intensities (present and estimated potential performances)

o SPS for CNGS: $4.4 \square 10^{13}$ and $7 \square 10^{13} \mathrm{ppp}$

o PS for CNGS: $3 \square 10^{13}$ and $4 \square 10^{13}$ ppp.

The LHC pilot beam is a "safety beam" to be used to establish circulating beam. The following supercycles have been assumed:

- LHC filling super-cycle:

o 1 LHC filling (flat porch for 4 PS injections), nominal length $\geq 21.6 \mathrm{~s}$ 
- LHC pilot super-cycle:

o 1 LHC pilot + 2 CNGS, nominal length: $22.8 \mathrm{~s}$

- CNGS\&SFT super-cycle:

o 3 CNGS + 1 SFT + 1 MD (Machine Development), nominal length: $34.8 \mathrm{~s}$.

Without any improvement, the basic requests cannot be met, especially for the ISOLDE and SPS users (Table 2 illustrates a case where priority is given to CNGS, resulting in a low flux for COMPASS). Moreover, the large level of beam loss associated with the CNGS operation in the PS will cause irradiation of PS equipment with detrimental consequences on reliability and maintenance.

Table 2: Proton flux in 2007 without improvement to the accelerators ("pot" stands for protons on target).

\begin{tabular}{|c|c|c|}
\cline { 2 - 3 } \multicolumn{1}{c|}{} & Available flux & Basic user’s requests \\
\hline $\begin{array}{c}\text { CNGS flux } \\
\left(\square 10^{19} \text { pot/year }\right)\end{array}$ & 4.4 & 4.5 \\
\hline $\begin{array}{c}\text { COMPASS spills } \\
\left(\square 10^{5} / \text { year }\right)\end{array}$ & 1.9 & 7.2 \\
\hline $\begin{array}{c}\text { ISOLDE flux }(\mu \mathrm{A}) \\
{[\text { pulses/hour] }}\end{array}$ & $\begin{array}{c}1.75 \\
{[1215]}\end{array}$ & $\begin{array}{c}1.9 \\
{[1350]}\end{array}$ \\
\hline $\begin{array}{c}\text { PS East area spills } \\
\left(\square 10^{6} / \text { year }\right)\end{array}$ & 1.5 & 1.3 \\
\hline $\begin{array}{c}\mathrm{nToF} \text { flux } \\
\left(\square 10^{19} \text { pot/year }\right)\end{array}$ & 1.7 & 1.5 \\
\hline
\end{tabular}

\section{Recommendations of the HIP WG}

Having considered the possible solutions, the following recommendations are made:

- In the short term, define in 2004 and start in 2005 three projects: (i) a new multi-turn PS extraction, to reduce beam loss and activation, (ii) an increased intensity in SPS for CNGS (implications in all machines), (iii) the reduction from $1.2 \mathrm{~s}$ to $0.9 \mathrm{~s}$ of the PSB repetition time.

- In the medium term, design a new linac ("Linac4") for the replacement of Linac2, with the goal of preparing for a decision of construction at the end of 2006.

- In the long term, prepare for a decision concerning the optimum future accelerators by pursuing the study of a Superconducting Proton Linac and by exploring alternative scenario for the LHC upgrade.

The estimated performance resulting from the implementation of the short and medium term measures is shown in Table 3. Numbers without parenthesis can be obtained by giving the highest priorities to CNGS. Conversely, the numbers in parenthesis are achieved by giving priority to COMPASS and limiting CNGS to its basic request. These improvements, and especially Linac4, should allow reaching the "ultimate" luminosity presently foreseen in the LHC by providing a proton beam with the "ultimate" characteristics.

\section{Status of implementation}

The three proposed studies have been approved and project proposals including requests for resources will be submitted at the end of 2004.

Developments for "Linac4" [3] are taking place with the support of the European Union and of the International Science and Technology Center (Moscow). CERN management confirmed recently its commitment to decide in 2006, with a possible construction starting in 2007.

Table 3: Proton flux in 2010 after implementation of the improvements recommended by the HIP WG.

\begin{tabular}{|c|c|c|}
\hline & Estimated flux & Basic user's request \\
\hline $\begin{array}{c}\text { CNGS flux } \\
\text { ( } \square 10^{19} \text { pot/year) }\end{array}$ & $7.5(4.5)$ & 4.5 \\
\hline $\begin{array}{c}\text { COMPASS spills } \\
\left.\text { ( } \square 10^{5} / \text { year }\right)\end{array}$ & $3.3(5.6)$ & 7.2 \\
\hline $\begin{array}{c}\text { ISOLDE flux }(\mu \mathrm{A}) \\
\text { [pulses/hour] }\end{array}$ & $\begin{array}{c}6.4 \\
{[2240]}\end{array}$ & $\begin{array}{c}1.9 \\
{[1350]}\end{array}$ \\
\hline $\begin{array}{c}\text { PS East area spills } \\
\text { ( } \square 10^{6} / \text { year) }\end{array}$ & 1.5 & 1.3 \\
\hline $\begin{array}{c}\text { nToF flux } \\
\text { ( } \square 10^{19} \text { pot/year) }\end{array}$ & 1.6 & 1.5 \\
\hline
\end{tabular}

A Superconducting Proton Linac ("SPL") $[4,5]$ is being studied as a possible replacement for the PSB as well as a proton driver for a second generation ISOL facility ("EURISOL") [6] and for neutrino physics [7]. An updated Conceptual Design Report will be published in 2005. The investigation of the alternative solutions based on a synchrotron is highly desirable but resources at CERN are presently not available.

\section{LONG TERM POSSIBILITIES}

Decisions for the long term (beyond 2010) have to take into account (i) the need to replace the aging accelerators, (ii) the plans for upgrading the LHC and (iii) the future physics programmes.

Some scenarios have already been proposed for the LHC upgrade, and, for example, the interest of a new $1 \mathrm{TeV}$ injector replacing the SPS has been mentioned [8] which would probably have to be coupled with a new $50 \mathrm{GeV}$ synchrotron replacing the PS. However, a detailed study is still needed to compare the different possibilities and draw all conclusions.

Concerning the future physics programmes, the HIP working group has already identified a number of possibilities envisaged by nuclear and neutrino physicists that could be satisfied with a multi-MW/ few GeV proton driver like the SPL.

Since then, requests for physics with kaons and muons have shown the potential interest of a multi-MW proton source at $30-50 \mathrm{GeV}$.

Although, in principle, all these requirements can be simultaneously satisfied, the needed resources are rather large and the consequences so important for the complex 
Table 4: Possible improvement to the accelerator complex

("RCS”=Rapid Cycling Synchrotron, "HEP”=High Energy Physics, “mMW”=multi-MW, "SC”=Superconducting)

\begin{tabular}{|c|c|c|c|c|c|c|}
\hline \multirow[b]{2}{*}{$\begin{array}{c}\text { Present } \\
\text { accelerator }\end{array}$} & \multirow[b]{2}{*}{$\begin{array}{l}\text { Replacement } \\
\text { accelerator }\end{array}$} & \multirow[b]{2}{*}{ Improvement } & \multicolumn{4}{|c|}{ INTEREST FOR } \\
\hline & & & $\begin{array}{c}\text { LHC } \\
\text { upgrade }\end{array}$ & $\begin{array}{l}\text { Neutrino } \\
\text { physics } \\
\text { beyond } \\
\text { CNGS } \\
\end{array}$ & $\begin{array}{l}\text { Radio-active } \\
\text { ion beams } \\
\text { beyond } \\
\text { ISOLDE } \\
\end{array}$ & $\begin{array}{c}\text { Physics } \\
\text { with kaons } \\
\text { and muons }\end{array}$ \\
\hline Linac2 & Linac4 & $\begin{array}{l}50 \rightarrow 160 \mathrm{MeV} \\
\mathrm{H}^{+} \rightarrow \mathrm{H}^{-}\end{array}$ & + & 0 (if alone) & 0 (if alone) & 0 (if alone) \\
\hline \multirow{3}{*}{ PSB } & $\begin{array}{c}2.2 \mathrm{GeV} \text { RCS for } \\
\text { HEP }\end{array}$ & $\begin{array}{l}1.4 \rightarrow 2.2 \mathrm{GeV} \\
10 \rightarrow 250 \mathrm{~kW} \\
\text { Brightness } \times 2\end{array}$ & + & 0 (if alone) & + & 0 (if alone) \\
\hline & $\begin{array}{c}2.2 \mathrm{GeV} / \mathrm{mMW} \\
\mathrm{RCS}\end{array}$ & $\begin{array}{l}1.4 \rightarrow 2.2 \mathrm{GeV} \\
0.01 \rightarrow 4 \mathrm{MW} \\
\text { Brightness } \times 2\end{array}$ & + & $\begin{array}{c}++ \\
\text { for super-beam } \\
\text { and beta-beam }\end{array}$ & $\begin{array}{c}+ \\
\text { (too short beam } \\
\text { pulse) }\end{array}$ & 0 (if alone) \\
\hline & $\begin{array}{c}2.2 \mathrm{GeV} / 50 \mathrm{~Hz} \\
\mathrm{SPL}\end{array}$ & $\begin{array}{c}1.4 \rightarrow 2.2 \mathrm{GeV} \\
0.01 \rightarrow 4 \mathrm{MW} \\
\text { Brightness } \times 2\end{array}$ & + & $\begin{array}{c}+++ \\
\text { for super-beam } \\
\text { and beta-beam }\end{array}$ & +++ & 0 (if alone) \\
\hline \multirow{2}{*}{ PS } & $\begin{array}{c}50 \mathrm{GeV} \text { SC PS for } \\
\text { HEP }\end{array}$ & $\begin{array}{l}26 \rightarrow 50 \mathrm{GeV} \\
\text { Intensity } \times 2 \\
\text { Brightness } \times 2 \\
\end{array}$ & ++ & 0 (if alone) & 0 & + \\
\hline & $50 \mathrm{GeV} / 5 \mathrm{~Hz} \mathrm{RCS}$ & $\begin{array}{l}26 \rightarrow 50 \mathrm{GeV} \\
0.1 \rightarrow 4 \mathrm{MW} \\
\text { Brightness } \times 2\end{array}$ & ++ & ++ & 0 & +++ \\
\hline SPS & $\begin{array}{c}1 \mathrm{TeV} \text { SC } \\
\text { Synchrotron }\end{array}$ & $\begin{array}{l}0.45 \rightarrow 1 \mathrm{TeV} \\
\text { Intensity } \times 2 \\
\text { Brightness } \times 2\end{array}$ & +++ & $?$ & 0 & +++ \\
\hline
\end{tabular}

that priorities have to be established, at least to plan for a staged realization.

\section{Analysis}

Assuming that accelerators are replaced not only to improve reliability but also to improve the characteristics of the beam (energy, intensity, brightness...), the replacement of a given machine should be coupled with the change of its injector(s). Therefore the decision process begins at low energy end and progressively covers all the energy range. The possibilities and the benefits for the different families of users are shown in Table 4. The comments and numbers are indicative and subject to evolution. Possible schemes are presented below but would require further and detailed studies before realistic proposals could be made.

\section{Linac2}

Linac4 is a necessary first step, whatever the choices for the other machines, because it is designed to be compatible with the most demanding applications. For the higher energy accelerators, the choice is more open and depends upon the physics programmes.

\section{$P S B$}

If a second generation ISOL-type facility has to be hosted at CERN, the SPL is the ideal solution, which can also be used for all scenarios of neutrino physics (super- beam, beta-beam and neutrino factory). The SPL would also be an outstanding replacement of the PSB for the following accelerators serving high energy physics experiments. Once the precise goals of such a multi-MW/ few $\mathrm{GeV}$ driver are defined, the possibility of a solution based on Rapid Cycling Synchrotron (RCS) should be analysed and the comparison should be made with the SPL.

If no experimental programme is approved that needs such a beam power at a few $\mathrm{GeV}$, the most economical solution is to build a small size RCS able to fill the PS or its successor in 4 or 8 pulses. Most beam pulses would be available for other users, which could be of interest for radio-active ion production, although not at the level required by EURISOL ( $200 \mathrm{~kW}$ of beam power instead of $4 \mathrm{MW})$.

\section{PS}

The low energy of the PS beam presently limits the SPS performance and the situation will be worse if the SPS is replaced with a superconducting synchrotron reaching $1 \mathrm{TeV}$. The present estimate is that the successor of the PS should deliver beam at approximately $50 \mathrm{GeV}$. If a multiMW beam power is needed at that energy, a Rapid Cycling Synchrotron should be considered. It would be a very challenging machine, surpassing the most ambitious synchrotron presently in construction in Japan [9]. Moreover, it would probably lack the flexibility of the 
present PS which would then have to be maintained in operation for the needs of heavy ions for LHC and slow ejection to the East area.

If multi-MW of beam power at $50 \mathrm{GeV}$ is not needed, a synchrotron using superconducting magnets could replace the PS. The magnets in development for the needs of SIS100 at GSI could be of interest for that application [10]. Indeed, the key technological item for such a synchrotron will be a fast pulsing SC dipole reaching a 4 to 6 Tesla field in a 2 to 3 second ramp [10-16]. The new process of multi-turn ejection which minimizes beam loss should be used.

\section{SPS}

Injection at $1 \mathrm{TeV}$ in the LHC would drastically ease operation with the present magnets, open some interesting possibilities for upgrading the luminosity beyond the ultimate value, and would even be necessary for an LHC energy upgrade. Other users could also be interested, provided the new machine is capable of slow ejection. The developments taking place in GSI for the superconducting magnets of SIS300 could be exploited [10].

LHC

Extracted proton beams at $7 \mathrm{TeV}$ are a potential field of investigation. Physics case of interest should however be more deeply discussed.

\section{ACKNOWLEDGMENTS}

We acknowledge the support of the European Community-Research Infrastructure Activity under the FP6 "Structuring the European Research Area" programme (CARE, contract number RII3-CT-2003506395).

\section{REFERENCES}

[1] http://ab-div.web.cern.ch/ab-div/Projects/hip/

[2] M. Benedikt, K. Cornelis, R. Garoby, E. Metral, F. Ruggiero, M. Vretenar, "Report of the High Intensity Protons Working Group”, CERN-AB-2004-022 OP/RF.

[3] R. Garoby, K. Hanke, A. Lombardi, C. Rossi, M. Vretenar, "Design of the Linac4, a new Injector for the CERN Booster", to be published in the proceedings of the LINAC2004 conference, August 16-20, Lubeck, Germany.

[4] http://ps-div.web.cern.ch/ps-div/SPL_SG/

[5] M. Vretenar (editor), "Conceptual Design of the SPL, A High Power Superconducting $\mathrm{H}^{-}$Linac at CERN”, CERN 2000-012.

[6] http://www.ganil.fr/eurisol/

[7] http://muonstoragerings.web.cern.ch/muonstoragerings I

[8] O. Bruning et al., "LHC Luminosity and Energy Upgrade: A Feasibility Study”, LHC Project Report 626.

[9] http://j-parc.jp/

[10]http://www.gsi.de/zukunftsprojekt/beschleunigeranla ge_e.html

[11] http://www-aix.gsi.de/annrep2001/files/171.pdf

[12] G. Moritz et al. "Towards Fast-Pulsed

Superconducting Synchrotron Magnets” PAC 2001.

[13] A. Kovalenko et al. "Superconducting Fast-Cycling

Dipole magnets for the GSI Future Accelerator Facility”,

EPAC 2002.

[14]M. N. Wilson "Design Study on Superconducting Cos teta Magnets for a Fast Pulsed Synchrotron” MT 17, 2002

[15] A. D. Kovalenko http://lhe.jinr.ru/JINR-GSI

[16] A. Bezaguet et al, “A Pulsed Superconducting Magnet for a Static Magnetic Refrigerator Operating between $1.8 \mathrm{~K}$ and $4.5 \mathrm{~K}$ ”, LHC Note 241, CERN AT 9329 (CR). 